\title{
Decreased mean platelet volume predicts poor prognosis in metastatic colorectal cancer patients treated with first-line chemotherapy: results from $\mathrm{mCRC}$ biomarker study
}

Jinjia Chang ${ }^{1,2 \dagger}\left(\mathbb{D}\right.$, Guangyi Lin $^{3 \dagger}$, Min Ye ${ }^{4 \dagger}$, Duo Tong ${ }^{1,2}$, Jing Zhao ${ }^{1,2}$, Dan Zhu ${ }^{1,2}$, Qihe Yu ${ }^{1,2}$, Wen Zhang ${ }^{1,2 \dagger}$ and Wenhua Li $i^{1,2^{*}+}$

\begin{abstract}
Background: Metastatic colorectal cancer (mCRC) is a major cause of death of malignant tumor and the valuable prognostic biomarker for chemotherapy is crucial in decreasing mortality. Previous studies have proved the prognostic value of the mean platelet volume (MPV) in survival of primary operable CRC patients. However, the prognostic impact of MPV in MCRC is still unclear. In this study, we aimed to clarify the prognostic role of MPV in mCRC undergoing standard first-line chemotherapy.

Methods: From January 2012 to December 2016, we conducted a retrospective clinical study included 264 mCRC patients (NCT03532711). All the enrolled patients received the standard oxaliplatin-based or irinotecan-based chemotherapy. The association between the baseline MPV and clinicopathological features were examined.

Results: Univariate analysis revealed that decreased MPV, the platelet counts (PLT), platelet-to-lymphocyte ratio $(P L R)$ and the platelet crit $(P C T)$ were significantly associated with inferior overall survival $(O S)(p<0.05)$. On multivariate analysis, elevated PLR was significant prognostic factors for OS, with hazard ratios of (HR:1.006, 95\% Cl:1. $001-1.011, p=0.01)$ while MPV was not, respectively $(p<0.05)$.
\end{abstract}

Conclusions: Our study demonstrated that the baseline MPV level may act as a predictive factor for survival in mCRC patients undergoing standard chemotherapy.

Trial registration: This study was retrospectively registered in date May the 20th 2018. The registration number (TRN) of this study was NCT03532711.

Keywords: MPV, Metastatic colorectal cancer, Chemotherapy, Biomarker

\footnotetext{
* Correspondence: whliiris@hotmail.com

${ }^{\dagger}$ Jinjia Chang, Guangyi Lin, Min Ye, Wen Zhang and Wenhua Li contributed equally to this work.

'Department of Medical Oncology, Shanghai Cancer Center, Fudan University, Shanghai, People's Republic of China

${ }^{2}$ Department of Oncology, Shanghai Medical College, Fudan University,

Shanghai 200032, China

Full list of author information is available at the end of the article
}

(c) The Author(s). 2019 Open Access This article is distributed under the terms of the Creative Commons Attribution 4.0 International License (http://creativecommons.org/licenses/by/4.0/), which permits unrestricted use, distribution, and reproduction in any medium, provided you give appropriate credit to the original author(s) and the source, provide a link to the Creative Commons license, and indicate if changes were made. The Creative Commons Public Domain Dedication waiver (http://creativecommons.org/publicdomain/zero/1.0/) applies to the data made available in this article, unless otherwise stated. 


\section{Background}

Colorectal cancer (CRC) is the third most common malignancies in males and second most common in females worldwide. The fatality rate ranks the fourth and the third for males and females, respectively [1]. Standard chemotherapy remains the most effective therapy for patients with metastatic colorectal cancer (mCRC) [2]. However, some patients do not benefit from chemotherapy but are exposed to the adverse effects [3]. Therefore, there is an urgency to develop reliable prognostic biomarkers for mCRC patients receiving standard chemotherapy.

In our previous study, we set up a clinical trial to evaluate potential biomarker of $\mathrm{mCRC}$ patients undergoing routine chemotherapy (mCRC biomarker study, https://www.clinicaltrials.gov, NCT03532711). One of our published paper indicated that patients carrying several SNP combinations may benefit more from the first-line chemotherapy such as FOLFOX/XELOX regimen. Our results suggested that the combination of SNPs may predict the therapeutic efficacy of the first-line chemotherapy for mCRC patients [4]. In the current study, we aimed to identify prognostic mCRC biomarkers that are low-cost and easily obtainable via routine blood counts.

It is well known that platelet activation acted as an active role in cancer progression and metastasis [5]. MPV which measures the average size of platelets in the blood might act as a marker of platelet activation [6-8]. MPV and PLT count are two main characteristics to evaluate platelet activation. Previous studies have revealed that MPV or its related factors such as PLT or PDW were crucial in many malignancies progression [9-12]. Notably, most of the research focused on early stage tumor or primarily operable cancer, few of them discussed the value of MPV in predicting late stage cancer patients' outcome [12, 13]. For example, the predictive value of PLT combined with MPV for overall survival in mCRC undergoing chemotherapy has not been yet investigated.

Herein, by conducting a clinical trial (NCT03532711), we aimed to investigate the effect of MPV levels on pathological factors as well clinical outcome of mCRC patients undergoing routine chemotherapy.

\section{Methods}

\section{Study description}

A retrospective observational clinical study was conducted to find the biomarkers in chemotherapy regimens for first-line chemotherapy for mCRC (NCT03532711). Patients with histopathologically confirmed mCRC who had at least one measurable lesion were enrolled. Two hundred sixty-four mCRC patients were enrolled in this clinical study and assigned to receive standard first-line chemotherapy such as FOLFOX/XELOX/FOLFIRI regimen according to the investigators' suggestion. The primary end point was objective response rate. Secondary end points were overall survival and progression-free survival. All patients collected pre-treatment complete blood cell counts. Treatment efficacy was regularly evaluated, and the best response efficacy was recorded according to the RECIST 1.1. This retrospective study was approved by the Ethical Committees of Fudan University Shanghai Cancer Center (Ethics Number: 1203108-10).

\section{Biochemical measurement}

We collected the peripheral venous blood sampling from each patient at the baseline of therapy. Whole blood samples were collected in EDTA-containing tubes for validation of platelet counts, white blood cell (WBC) and hemoglobin.

\section{Statistical analysis}

We used the Receiver-operating characteristics (ROC) curve to select the optimum cut-of value of MPV. The Student's t-test was used to determine the significance of between-group differences were determined. The KaplanMeier method was used to describe analysis of survival curves. Independent factors were identified by multivariate Cox proportional hazards modeling. A value of $p<0.05$ was considered of significance. All statistical analyses were conducted using SPSS Statistics version 22.0.

\section{Results}

A total of 264 metastatic mCRC patients were enrolled in this clinical study (NCT03532711). One hundred seven patients $(40.5 \%)$ were women and 157 patients (59.5\%) were men, and the median age was 55.5 years old. $165(62.5 \%)$ patients suffered from colon cancer while 99 (37.5\%) patients were with rectal cancer. For tumor metastatic pattern, 146 of 264 patients (55.3\%) were with synchronous metastasis and the rest 118 patients were with metachronous metastasis.

MPV level were recorded in all the patients at baseline. As shown in Additional file 1: Figure S1, 108 patients were out of normal range while 156 patients were in the normal range. We used a ROC curve to determine the optimal cut-off value of MPV. As a result, an MPV of $9.75 \mathrm{fL}$ yielded maximum combined sensitivity and specificity (see Fig.1). It is showed that MPV predicted cancer prognosis with a sensitivity of 0.625 and a specificity of 0.272 (AUC $=0.473,95 \% \mathrm{CI}: 0.402-0.544, p=$ $0.459)$. Therefore, we could clearly classify the patients into two independent groups: patients with MPV $\geq 9.75$ $\mathrm{fL}$ and patients with MPV $<9.75 \mathrm{fL}$. In this study, 173 (65.6\%) patients were with MPV $\geq 9.75 \mathrm{fL}$ and $91(34.4 \%)$ patients were with $\mathrm{MPV}<9.75 \mathrm{fL}$.

Table 1 and Table 2 showed the relationship between MPV and clinical characteristics. Some of the clinicolaboratory characteristics (PLT, PCT, PLR, hemoglobin, ANC, WBC, PLR, MPV/P, PDW/P and Efficacy) were 


\section{MPV}

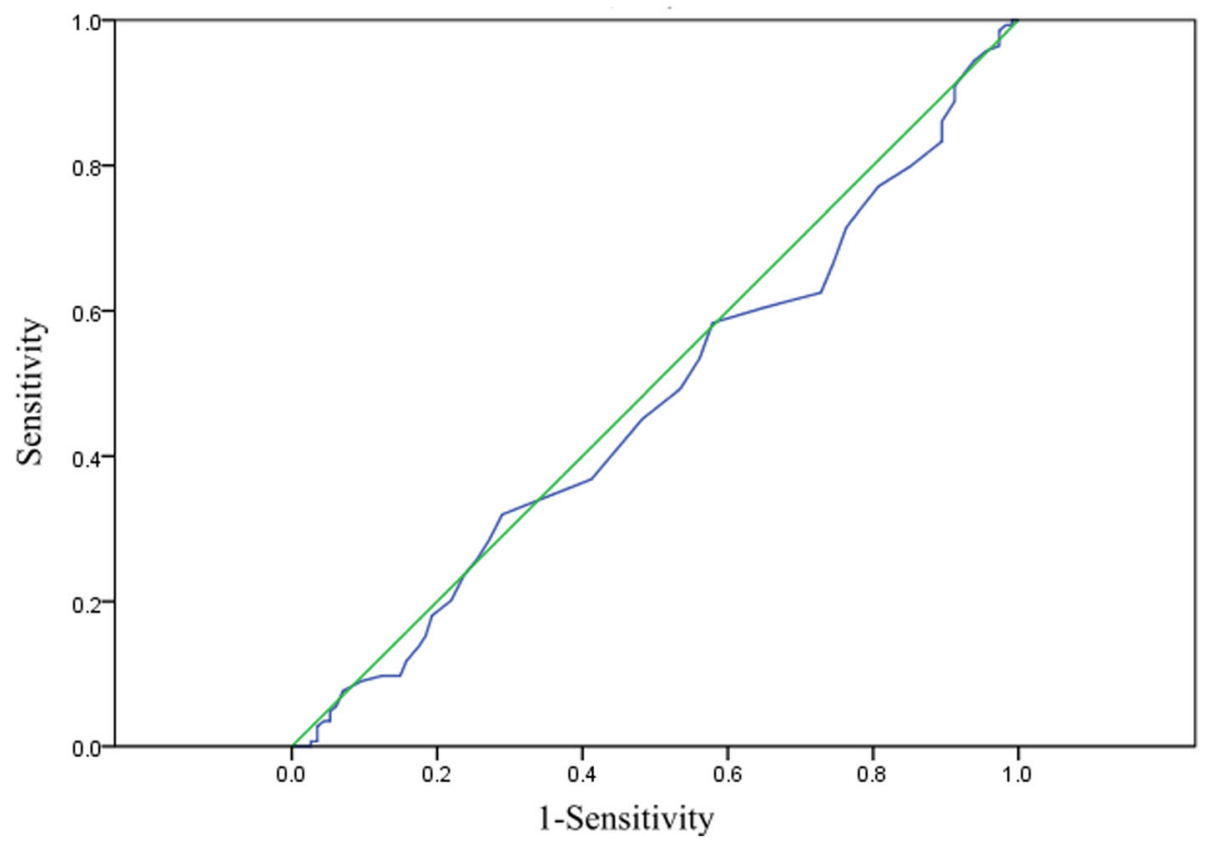

Fig. 1 Optimized cut-off was determined for MPV using standard ROC curve analysis

demonstrated closely correlated with MPV. The other factors including age, gender, location, regimen, histology and metastasis pattern showed no difference between the two groups.

The Kaplan-Meier method was used to describe analysis of survival curves. As is shown in Fig. 2, patients with low MPV levels had worse OS than those with high MPV levels (22.57 \pm 1.813 months vs. $18.37 \pm 2.132$ months, $p=0.047)$. Our results demonstrated that mCRC patients with decreased MPV level have an inferior outcome than those patients with high MPV level.

Analysis of variance was performed to clarify the relationship between MPV and ORR. The results showed that ORR was $39.6 \%$ in the patients with high MPV levels and $24.1 \%$ in the patients with low MPV levels. In the univariate analyses, MPV and several clinicolaboratory characteristics (gender, hemoglobin, PLT, WBC, ANC, PCT, PLR, NLR and efficacy) were associated with PFS or OS ( see Table 3 and Additional file 2: Table S1). Those characteristics with a $p$-value less than 0.1 were included in the multivariate analysis. As a result, WBC, ANC, PLR, PLT and efficacy were independent factors for OS (see Table 3 and Table 4).

\section{Discussion}

Altered MPV might be valuable prognostic biomarker for malignant tumor patient, however, MPV level and its relationship with the patient outcome remains unclear. For example, previous studies suggested that elevated MPV levels were proved significantly associated with better outcome in esophageal cancer [3], breast cancer [13] while other research indicated that reduced MPV were associated with worse outcome in lung cancer [14], renal cancer [15] and bladder cancer [16]. As for colorectal cancer, the patients with elevated MPV level, compared with those with normal level, were found had worse overall survival [17]. Notably, to our best of knowledge, there lacks the analysis of the survival impact of MPV in patients with mCRC treated with first-line chemotherapy. In this study, we aimed to analysis the correlation between pretreatment MPV level and overall survival in patients with metastatic CRC undergoing the first line chemotherapy regimens.

In order to study the issue by a more precise way, we conducted a prospective clinical study to characterize and evaluate biomarkers of chemotherapy in patients with $\mathrm{mCRC}$ in the first-line setting. Two hundred sixty-four patients were enrolled and they were treated with first line chemotherapy including FOLFOX/ XELOX/FOLFIRI. Our results showed that the group of patients with decreased MPV level had worse overall survival than the group with high MPV level (shown in Fig. 2). In addition, we performed univariate analysis and found that MPV, together with platelet count, PCT and PLR, were associated with OS. Unlikely, PLR but not MPV was demonstrated as an independent prognostic factor for mCRC undergoing chemotherapy. In contrast with our study, $\mathrm{Yu}$ et al reported that operative CRC patients with elevated MPV level had poor overall survival 
Table 1 Baseline characteristics of patients with mCRC according to MPV levels

\begin{tabular}{|c|c|c|c|c|}
\hline Variables & Total n (\%) & MPV $\geq 9.75$ & $M P V<9.75$ & $P$ value \\
\hline Age (years) & & & & 0.6329 \\
\hline$\leq 65$ & 209(79.2\%) & 135(78.0\%) & 74(81.3\%) & \\
\hline$>65$ & $55(20.8 \%)$ & $38(22.0 \%)$ & $17(18.7 \%)$ & \\
\hline Gender & & & & 0.9753 \\
\hline Male & $157(59.4 \%)$ & 103(59.5\%) & $54(59.3 \%)$ & \\
\hline Female & $107(40.6 \%)$ & $70(40.5 \%)$ & $37(40.7 \%)$ & \\
\hline Location & & & & 0.5950 \\
\hline Colon & $164(62.3 \%)$ & $106(61.3 \%)$ & $59(64.8 \%)$ & \\
\hline Rectum & 99 (37.7\%) & $67(38.7 \%)$ & $32(35.2 \%)$ & \\
\hline Regimen & & & & 0.3024 \\
\hline Oxaliplatin & $125(46.2 \%)$ & $86(49.7 \%)$ & $39(42.9 \%)$ & \\
\hline Irinotecan & $142(53.8 \%)$ & $87(50.3 \%)$ & $52(57.1 \%)$ & \\
\hline Histology & & & & 0.3593 \\
\hline adenocarcinoma & 239(90.5\%) & $161(93.1 \%)$ & $78(85.7 \%)$ & \\
\hline mucinous adenocarcinoma & $15(5.7 \%)$ & $7(4.0 \%)$ & $8(8.8 \%)$ & \\
\hline signet-ring cell carcinoma & $4(1.5 \%)$ & $2(1.2 \%)$ & $2(2.2 \%)$ & \\
\hline NK & $6(2.3 \%)$ & $3(1.7 \%)$ & $3(3.3 \%)$ & \\
\hline Metastasis pattern & & & & 0.4852 \\
\hline simultaneous & $146(55.3 \%)$ & $93(53.8 \%)$ & $53(58.2 \%)$ & \\
\hline metachronous & $118(44.7 \%)$ & $80(46.2 \%)$ & $38(41.7 \%)$ & \\
\hline Efficacy & & & & 0.0166 \\
\hline$C R+P R$ & $84(31.8 \%)$ & $65(37.6 \%)$ & 19(20.9\%) & \\
\hline SD & $132(50.0 \%)$ & $81(46.8 \%)$ & $51(56.0 \%)$ & \\
\hline PD & $27(10.2 \%)$ & 18(10.4\%) & $9(9.9 \%)$ & \\
\hline NK & $21(8.0 \%)$ & $9(5.2 \%)$ & $12(13.2 \%)$ & \\
\hline
\end{tabular}

Abbreviations: NK not known, $C R$ complete response, $S D$ stable disease, $P D$ progression disease

Table 2 Association of MPV with the clinicolaboratory characteristics of patients with $\mathrm{mCRC}$

\begin{tabular}{llll}
\hline Variables & MPV $\geq 9.75$ & MPV $<9.75$ & P value \\
\hline PLT $\left(\times 10^{9} / L\right)$ & 226.82 & 287.81 & 0.000 \\
PCT & 0.25 & 0.27 & 0.079 \\
PDW $(\%)$ & 13.11 & 10.21 & 0.000 \\
Hemoglobin $\left(\leq 120 \times 10^{9} / L\right.$ vs. $\left.>120 \times 10^{9} / L\right)$ & $18.23 \pm 1.49$ & $25.33 \pm 2.637$ & 0.006 \\
ANC $\left(\times 10^{9} / L\right)$ & 4.53 & 5.25 & 0.016 \\
WBC $\left(\times 10^{9} / L\right)$ & 6.67 & 7.46 & 0.017 \\
PLR & 173.48 & 209.46 & 0.006 \\
NLR & 3.579 & 3.89 & 0.353 \\
MPV/P & 0.06 & 0.03 & 0.000 \\
PDW/P & 0.07 & 0.04 & 0.000 \\
\hline A & & &
\end{tabular}

Abbreviations: $P L T$ platelet count, $P C T$ platelet crit, $P D W$ platelet distribution width, WBC white blood cell, $A N C$ neutrophil cell, MPV mean platelet volume, NLR neutrophil-to-lymphocyte ratio, $P L R$ platelet-to-lymphocyte ratio, MPV/P MPV-to-platelet ratio, PDW/P PDW-to-platelet ratio 

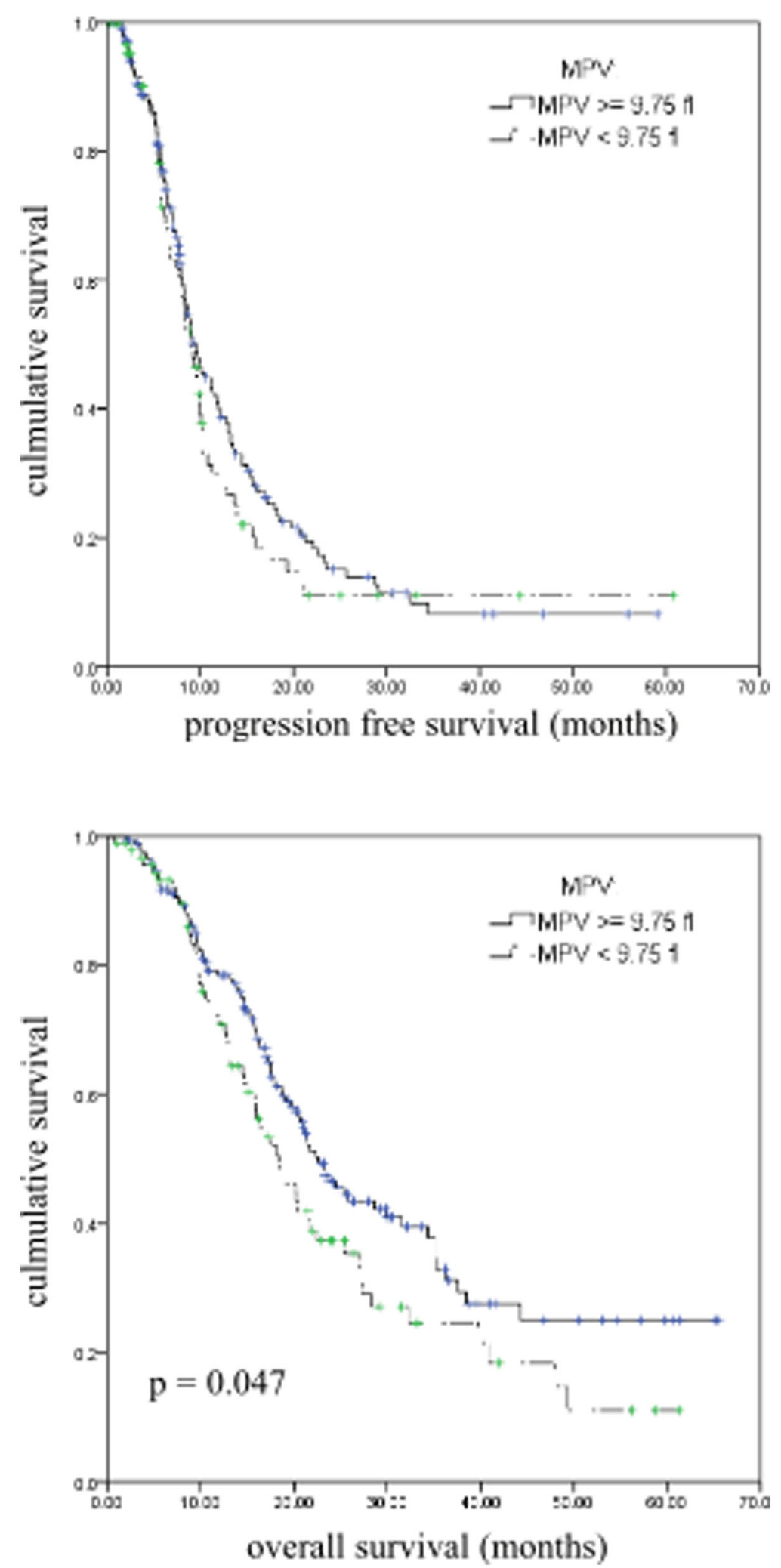

Fig. 2 Influence of MPV levels on overall survival and progression free survival by Kaplan-Meier analyses. The Kaplan-Meier survival curve with log-rank analysis of OS showed statistical significance between curves of patients with MPV low level and high level in mCRC biomarker study (log-rank test) 
Table 3 Results of univariate analysis of overall survival in patients with mCRC

\begin{tabular}{llll}
\hline Variables & Hazard ratio & $95 \% \mathrm{Cl}$ & P-value \\
\hline Age (years) $(\geq 65$ versus $<65)$ & 0.918 & $0.56-1.261$ & 0.400 \\
Gender & 1.432 & $1.037-1.977$ & 0.029 \\
MPV $(\geq 9.75 \mathrm{fL}$ versus $<9.75 \mathrm{fL})$ & 1.398 & $1.005-1.946$ & 0.047 \\
WBC & 1.082 & $1.018-1.150$ & 0.011 \\
ANC & 1.084 & $1.016-1.157$ & 0.015 \\
Lymphocyte & 0.861 & $0.64-1.157$ & 0.320 \\
Hemoglobin $\left(\leq 120 \times 10^{9} / \mathrm{L}\right.$ vs. $\left.>120 \times 10^{9} / \mathrm{L}\right)$ & 0.630 & $0.453-0.87$ & 0.006 \\
PLT & 1.002 & $1-1.003$ & 0.018 \\
PCT & 4.234 & $1.17-15.322$ & 0.028 \\
PDW & 0.961 & $0.901-1.026$ & 0.234 \\
PLR & 1.003 & $1-1.004$ & 0.002 \\
NLR & 1.066 & $1.007-1.128$ & 0.028 \\
Efficacy & 1.529 & $1.285-1.820$ & 0.000 \\
\hline A & & &
\end{tabular}

Abbreviation: see Table 1 and Table 2

compared with those with normal level. Our results indicated that the situation may be different in late-stage CRC compared with operable CRC [17].

The mechanisms underlying the association between MPV and $\mathrm{mCRC}$ patient outcome in is not entirely clear. MPV is an initial symbol of activated platelets [18]. Decreased MPV could be recognized as elevated expression of large platelets in inflammatory states [19]. Large platelets could release a variety of pro-inflammatory cytokines such as interleukin-6 (IL-6), which may improve tumor progression and metastasis [20]. It is well known that there is a confirm linkage between CRC and chronic inflammatory [21]. Several pre-treatment inflammatory indexes such as PLR and NLR were probed as predictors of prognosis and treatment efficacy in patients with metastatic colorectal cancer mCRC [22]. This may explain the reason that decreased MPV may result in mCRC tumor progression and shorter overall survival.
Our research has several limitations. First, our clinical study was a single-center retrospective study and more ethnic groups are warranted to confirm our results. Second, the mechanism of MPV in mCRC treated with chemotherapy should be further illustrated. For example, further investigation could be performed to clarify the association of decreased MPV and chemotherapy regimens such as oxaliplatin or fluorouracil.

\section{Conclusions}

In conclusion, MPV and its related factor PLR were proved in the current study to act as a prognostic biomarker for mCRC patients undergoing first-line chemotherapy. Future studies need to be developed to study the underlying mechanism of MPV in mCRC progression.

Table 4 Results of multivariate analysis of overall survival in patients with mCRC

\begin{tabular}{llll}
\hline Variables & HR & $95 \%$ Cl & $P$-value \\
\hline Gender & 1.307 & $0.932-1.833$ & 0.121 \\
MPV $(\geq 9.75 \mathrm{fL}$ versus $<9.75 \mathrm{fL})$ & 1.117 & $0.752-1.658$ & 0.584 \\
WBC & 1.589 & $1.193-2.116$ & 0.002 \\
ANC & 0.679 & $0.470-0.980$ & 0.038 \\
Hemoglobin $\left(\leq 120 \times 10^{9} / \mathrm{L}\right.$ vs. $\left.>120 \times 10^{9} / \mathrm{L}\right)$ & 0.809 & $0.548-1.193$ & 0.285 \\
PLT & 0.995 & $0.991-1.000$ & 0.038 \\
PCT & 2.470 & $0.237-25.702$ & 0.449 \\
NLR & 0.921 & $0.749-1.132$ & 0.436 \\
PLR & 1.007 & $1.003-1.012$ & 0.002 \\
Efficacy & 1.582 & $1.311-1.909$ & 0.000 \\
\hline
\end{tabular}

All variables with a $p$-value lower than 0.1 in univariate analysis were included in a multivariate statistical analysis 


\section{Additional files}

Additional file 1: Figure S1. MPV level distribution of 264 patients. (PNG $78 \mathrm{~kb}$ )

Additional file 2: Table S1. Results of univariate analysis of progression free survival in patients with mCRC. (DOCX $16 \mathrm{~kb}$ )

\section{Abbreviations}

HRs: Hazard ratios; mCRC: Metastatic colorectal cancer; MPV: Mean platelet volume; OS: Overall survival; PCT: Platelet crit; PLR: Platelet-to-lymphocyte ratio; PLT: Platelet counts; ROC: Receiver-operating characteristics; WBC: White blood cell

\section{Funding}

This work was supported financially by grants from the National Natural Science Foundation of China (NSFC81602081). None of the funding bodies played a role in data collection, analysis, or interpretation of data, the writing of the manuscript, or the decision to submit the manuscript for publication.

\section{Availability of data and materials}

Data sharing is not applicable to this article as no datasets were generated or analysed during the current study.

\section{Authors' contributions}

WHL, WZ and JJC conceived of the study. JJC and GYL contributed to intellectual content and interpretation of data from the study. DT and DZ abstracted clinical and histopathological data from medical charts. QHY performed the statistical analysis and drafted the manuscript. MY and JZ contributed to the statistical analysis and interpretation of the data. WHL, and WZ contributed with clinical expertise. All authors read and approved the final manuscript.

\section{Ethics approval and consent to participate}

This retrospective study was approved by the Ethical Committees of Fudan University Shanghai Cancer Center (Ethics Number: 1203108-10).

\section{Consent for publication}

Not applicable.

\section{Competing interests}

The authors declare that they have no competing interests.

\section{Publisher's Note}

Springer Nature remains neutral with regard to jurisdictional claims in published maps and institutional affiliations.

\section{Author details}

'Department of Medical Oncology, Shanghai Cancer Center, Fudan University, Shanghai, People's Republic of China. ${ }^{2}$ Department of Oncology, Shanghai Medical College, Fudan University, Shanghai 200032, China. ${ }^{3}$ Shanghai medical college, Fudan University, Shanghai 200032, China. ${ }^{4}$ Department of Pathology, Shanghai Cancer Center, Fudan University, Shanghai 200032, China.

Received: 11 October 2018 Accepted: 26 December 2018 Published online: 07 January 2019

\section{References}

1. Chua W, Kho PS, Moore MM, Charles KA, Clarke SJ. Clinical, laboratory and molecular factors predicting chemotherapy efficacy and toxicity in colorectal cancer. Crit Rev Oncol Hematol. 2011;79(3):224-50.

2. Fakih MG. Metastatic colorectal cancer: current state and future directions. J Clin Oncol. 2015;33(16):1809-24.

3. Zhang F, Chen Z, Wang P, Hu X, Gao Y, He J. Combination of platelet count and mean platelet volume (COP-MPV) predicts postoperative prognosis in both resectable early and advanced stage esophageal squamous cell cancer patients. Tumour Biol. 2016;37(7):9323-31.

4. Zhao J, Li W, Zhu D, Yu Q Zhang Z, Sun M, Cai S, Zhang W. Association of single nucleotide polymorphisms in MTHFR and ABCG2 with the different efficacy of first-line chemotherapy in metastatic colorectal cancer. Med Oncol. 2014:31(1):802.

5. Franco AT, Corken A, Ware J. Platelets at the interface of thrombosis, inflammation, and cancer. Blood. 2015;126(5):582-8.

6. Matsumura M, Chiba Y, Lu C, Amaya H, Shimomatsuya T, Horiuchi T, Muraoka $\mathrm{R}$, Tanigawa N. Platelet-derived endothelial cell growth factor/thymidine phosphorylase expression correlated with tumor angiogenesis and macrophage infiltration in colorectal cancer. Cancer Lett. 1998;128(1):55-63.

7. Tsuji T, Sawai T, Yamashita H, Takeshita H, Nakagoe T, Shindou H, Fukuoka H, Yoshinaga M, Hidaka S, Yasutake T, et al. Platelet-derived endothelial cell growth factor expression is an independent prognostic factor in colorectal cancer patients after curative surgery. Eur J Surg Oncol. 2004;30(3):296-302.

8. Denizot Y, Truffinet V, Bouvier S, Gainant A, Cubertafond P, Mathonnet M. Elevated plasma phospholipase A2 and platelet-activating factor acetylhydrolase activity in colorectal cancer. Mediat Inflamm. 2004;13(1):53-4.

9. Takeuchi H, Abe M, Takumi Y, Hashimoto T, Kobayashi R, Osoegawa A, Miyawaki M, Okamoto T, Sugio K. The prognostic impact of the platelet distribution width-to-platelet count ratio in patients with breast cancer. PLoS One. 2017;12(12):e0189166.

10. Kaito K, Otsubo H, Usui N, Yoshida M, Tanno J, Kurihara E, Matsumoto K Hirata R, Domitsu K, Kobayashi M. Platelet size deviation width, platelet large cell ratio, and mean platelet volume have sufficient sensitivity and specificity in the diagnosis of immune thrombocytopenia. $\mathrm{Br} J$ Haematol. 2005:128(5):698-702

11. Gao L, Zhang H, Zhang B, Zhang L, Wang C. Prognostic value of combination of preoperative platelet count and mean platelet volume in patients with resectable non-small cell lung cancer. Oncotarget. 2017;8(9): 15632-41.

12. Li N, Yu Z, Zhang X, Liu T, Sun YX, Wang RT, Yu KJ. Elevated mean platelet volume predicts poor prognosis in colorectal cancer. Sci Rep. 2017;7(1):10261.

13. Gu M, Zhai Z, Huang L, Zheng W, Zhou Y, Zhu R, Shen F, Yuan C. Pretreatment mean platelet volume associates with worse clinicopathologic features and prognosis of patients with invasive breast cancer. Breast Cancer. 2016;23(5):752-60.

14. Inagaki N, Kibata K, Tamaki T, Shimizu T, Nomura S. Prognostic impact of the mean platelet volume/platelet count ratio in terms of survival in advanced non-small cell lung cancer. Lung Cancer. 2014:83(1):97-101.

15. Yun ZY, Zhang X, Liu YS, LiU T, Liu ZP, Wang RT, Yu KJ. Lower mean platelet volume predicts poor prognosis in renal cell carcinoma. Sci Rep. 2017;7(1):6700.

16. Wang X, Cui MM, Xu Y, Liu L, Niu Y, Liu T, Liu ZP, Wang RT, Yu KJ. Decreased mean platelet volume predicts poor prognosis in invasive bladder cancer. Oncotarget. 2017;8(40):68115-22.

17. Yasui A, Naruse S, Yanaihara C, Ozaki T, Hoshino M, Mochizuki T, Daniel EE, Yanaihara N. Corelease of PHI and VIP by vagal stimulation in the dog. Am J Phys. 1987;253(1 Pt 1):G13-9.

18. Mezouar S, Frere C, Darbousset R, Mege D, Crescence L, Dignat-George F, Panicot-Dubois L, Dubois C. Role of platelets in cancer and cancerassociated thrombosis: experimental and clinical evidences. Thromb Res. 2016;139:65-76

19. Takeuchi H, Kawanaka H, Fukuyama S, Kubo N, Hiroshige S, Yano T. Comparison of the prognostic values of preoperative inflammation-based parameters in patients with breast cancer. PLoS One. 2017;12(5):e0177137.

20. Kaushansky K. Growth factors and hematopoietic cell fate. A new feature: controversies in hematology. Blood. 1998;92(2):345-4.

21. Riedl JM, Posch F, Moik F, Bezan A, Szkandera J, Smolle MA, Kasparek AK Pichler M, Stoger H, Stotz M, et al. Inflammatory biomarkers in metastatic colorectal cancer: prognostic and predictive role beyond the first line setting. Oncotarget. 2017;8(56):96048-61.

22. Passardi A, Scarpi E, Cavanna L, Dall'Agata M, Tassinari D, Leo S, Bernardini I, Gelsomino F, Tamberi S, Brandes AA, et al. Inflammatory indexes as predictors of prognosis and bevacizumab efficacy in patients with metastatic colorectal cancer. Oncotarget. 2016;7(22):33210-9. 